\title{
LA ALBAÑILERÍA SEVILLANA Y SU CARÁCTER ASISTENCIAL: EL HOSPITAL DE SAN ANDRÉS DE LOS ALBAÑILES
}

\author{
SEVILLIAN BRICKLAYERS TRADE AND ITS \\ ASSISTANCE CHARACTER: SAINT ANDREW'S \\ BRICKLAYERS HOSPITAL
}

\author{
David Gutiérrez De Los Santos \\ davidgutisantos@hotmail.com
}

\begin{abstract}
Para el completo conocimiento de los oficios de la construcción, deben atenderse los diferentes matices que conforman su estructura. Un colectivo que ha sufrido una suerte dispar en su estudio ha sido el de los albañiles, a pesar de protagonizar algunas de las más brillantes etapas de la arquitectura en Sevilla. En el presente artículo atenderemos a un primer momento fundacional, con la creación de la casa corporativa, que puede arrojar luz sobre un gremio fundamental para el desarrollo arquitectónico sevillano, y que debe enmarcarse en un estudio integrador del oficio de la albañilería hispalense.

Palabras clave: Albañiles, Gremio, Hospital de San Andrés, Reducción de los Hospitales, Arquitectura Sevillana.
\end{abstract}

To achieve a thorough knowledge of construction trades, we need to be familiar with the different specialities which make up this field. One group which has enjoyed contrasting fortunes is bricklayers, in spite of having played an important role in some of Seville's greatest eras of architecture. In this article, we will look at the early founding period, with the creation of the company's house, which can help throw some light on a trade which was essential to the architectural development of Seville, and which should be included in a comprehensive study of Sevillian bricklayers trade. tecture.

Keywords: Bricklayers, Trade, Saint Andrew's Hospital, Hospitals Reduction, Sevillian Archi-

\section{LA ALBAÑILERÍA SEVILLANA Y SU CARÁCTER ASISTENCIAL}

Dentro de las actividades desarrolladas por las corporaciones profesionales se encontraba la relacionada con el carácter asistencial hacia los integrantes del oficio. Como el resto de los gremios, el de los albañiles constituyó su propio hospital, que hacía las veces de casa gremial donde sus integrantes podían celebrar 
sus cabildos, así como la elección de cargos dirigentes y examinar a los oficiales que aspiraban a la obtención del grado de maestría. Además, se llevaban a cabo en él aspectos relacionados con la caridad, como el cuidado de huérfanos, asistencia a viudas y oficiales que habían llegado a la mendicidad ${ }^{1}$.

Su actividad se encuadraba en la corriente desarrollada en Sevilla desde finales de la Edad Media hasta el principio del Renacimiento, cuando fueron fundadas las grandes entidades hospitalarias, la mayoría de ellas patrocinadas por los Reyes Católicos. Del gran número de centros asistenciales existentes en la ciudad, llegando al casi centenar en el último tercio del siglo XVI, eran pocos los que desarrollaban la función de hospital tal como la entendemos en la actualidad.

Hasta aquel momento el sistema de hospitalidad en Sevilla se desarrollaba en torno a cuatro pilares fundamentales; los hospitales sanitarios, los asilos y orfanatos, los dependientes de hermandades o cofradías de caridad, y los centros adscritos a cofradías profesionales ligadas a los gremios ${ }^{2}$. Estos últimos habían nacido con la creación de las corporaciones ligadas a oficios, ya que los gremios sevillanos solían tener anexa una cofradía o hermandad, que estaba bajo la advocación del santo patrón, y que daba nombre al hospital. Las labores asistenciales desempeñadas por estos hospitales se limitaban al socorro de sus agremiados en caso de enfermedad, al auxilio de las viudas de los oficiales y a proporcionar enterramiento a los miembros fallecidos y sus familias. La casa-hospital cumplía la función de local para celebración de las reuniones profesionales, así como para los oficios religiosos y las fiestas que se desarrollaban como culto al santo patrón. La única vinculación de estos centros asistenciales con el término "hospital" estaba relacionada con el marcado carácter religioso que envolvía a las acciones desempeñadas por la cofradía, que tenían en la casa gremial su razón de ser, con lo que los cofrades solían denominarla hospital,

“...por ser como son todos sus cofrades cristianos viejos por particular devoción la pusieron nombre de hospital a la casa de sus juntas"3.

Se deduce de todo lo expuesto que dichas instituciones, dependientes de corporaciones profesionales, habían surgido de la voluntad de los gremios por disponer de centros propios donde poder celebrar sus cabildos y todos los asuntos relacionados con su profesión.

${ }^{1}$ CRUZ ISIDORO, Fernando: "Sobre los gremios de albañilería y carpintería en la Sevilla del XVII", Boletín del Seminario de Estudios de Arte y Arqueología, 67, 2001, p. 232.

2 Para un completo análisis del sistema hospitalario sevillano durante los siglos XVI, XVII y XVIII resulta imprescindible consultar la obra de, CARMONA GARCÍA, Juan Ignacio: El sistema de la hospitalidad pública en la Sevilla del Antiguo Régimen. Sevilla, 1979.

${ }_{3}$ Ibidem, p. 40. (Archivo de la Diputación Provincial de Sevilla, Amor de Dios, sección $1^{\mathrm{a}}$, legs. 33-36). 
Los ingresos que disfrutaban dichas fundaciones benéficas procedían de diversas fuentes, tales como arrendamiento de casas, tributos, donaciones de los cofrades e incluso el pago en especie, como podremos comprobar más tarde cuando abordemos el caso específico de San Andrés. Las limosnas, en la mayoría de las ocasiones, suponían el ingreso fundamental, ya que el resto de las vías de ingresos citadas eran insuficientes para soportar la mínima estructura requerida para su sustento y de su labor asistencial. Además, en numerosas ocasiones, recibían una dotación a cambio de la celebración de misas, memorias y remembranças.

Los gastos relacionados con el funcionamiento de dichas instituciones dependientes de cofradías gremiales estaban orientados fundamentalmente hacia las celebraciones de carácter religioso, lo que suponía un gasto importante en cera, aceite para las lámparas y todo lo necesario para la celebración de los diversos cultos, que en la mayoría de ocasiones estaba íntimamente ligado a los cabildos y fiestas propias de la profesión.

Otra de las partidas relacionadas con el gasto, estaba integrada por los salarios del escaso personal de mantenimiento de la institución; apenas un casero, encargado de la limpieza de las estancias, y en algunas ocasiones el del mayordomo o prioste, quien estaba a cargo de la administración y finanzas ${ }^{4}$.

\section{EL HOSPITAL DE SAN ANDRÉS DE LOS ALBAÑILES}

Para conocer el funcionamiento del hospital de San Andrés, apodado de los albañiles, poseemos una valiosa fuente de información en los documentos relacionados con la reducción de las instituciones hospitalarias ordenadas en 1587, que hasta el momento habían pasado desapercibidas para la historiografía local, en el caso que nos ocupa, con lo que podemos ofrecerlos aquí de forma inédita 5 . En dichos documentos se recoge el funcionamiento de la institución y algunos datos de suma importancia relacionados con su fundación.

El hospital de los profesionales de la albañilería estaba bajo la advocación de San Andrés, patrón del gremio, y se encontraba situado, tal y como señala la documentación consultada, en la collación del mismo nombre, frontero a las casas

\footnotetext{
${ }^{4}$ Ibíd., p. 88.

5 ADPS., Sección Hospitales y centros benéficos, Hospital del Amor de Dios, Legajo 1, expediente de reducción del "Hospital de San Andrés de los albañies", sin foliar. En el Apéndice Documental que se incluye al final de este artículo se encuentra el texto completo trascrito. El análisis de los documentos que se expone a continuación se basa en la documentación referida.
} 
de Pedro de Pineda, por una parte, y por la otra lindero a la casa que hacía esquina con la calle de la Venera y calle Real ${ }^{6}$, actuales José Gestoso y Orfila ${ }^{7}$.

A la hora de efectuar el expediente de reducción, se les conminaba a presentar documentación con la que pudiesen demostrar la legitimidad de sus propiedades. En ese momento, a 22 de noviembre de 1584, el albañil y prioste Martín Sánchez compareció ante la comisión con el firme propósito de frenar la reducción del hospital de San Andrés, siendo nombrado por el cabildo y los cofrades del gremio de la albañilería para tal fin. Debía dar cuenta de las propiedades y de los bienes raíces que poseía la institución, para lo cual exhibió los libros de cuentas y escrituras que estaban en posesión del gremio. Según estos documentos pudo probar en ese momento, mediante escritura pública, la propiedad de la casa que el hospital tenía, además de su situación y las lindes con las que era frontera. En ellos se dice que el conjunto hospitalario disponía de una capilla con un retablo y alta$\mathrm{res}^{8}$, donde se decía misa en las ocasiones que los agremiados se reunían para tratar temas profesionales en cabildo. Se especifica que el retablo con el que contaba la capilla estaba formado por diversas pinturas, ya que se dice que "dicho retablo es de pincel con ciertas figuras de santos" 9 .

En cuanto a la fundación de la casa-hospital se ofrece un dato de suma importancia en dicho expediente. Después de recibir juramento, el prioste del hospital, Martín Sánchez, fue preguntado acerca de la fundación de la casa gremial y exhortado a demostrar su titularidad. En ese momento exhibió un testamento, escrito en pergamino, según el cual María Pérez, viuda de Diego Fernández, había legado al gremio de albañiles de la ciudad una casa que podía ser utilizada para las reuniones de los oficiales, tanto para los cabildos ordinarios, como para la elección de alarifes y alcaldes del oficio, a cambio de una "remenbrança" que los cofrades estaban obligados a realizar en memoria de la donante. La fecha de dicha escritura de donación es muy significativa por lo temprano de su concesión, "a ocho dias del mes de mayo del año de mill y quatrocientos y un año", firmada ante el escribano público Gómez Pérez.

\section{${ }^{6}$ Ibidem. \\ ${ }^{7}$ COLLANTES DE TERÁN, Antonio: Diccionario histórico de las calles de Sevilla.} Sevilla, 1993, t. II, pp. 246 y 445.

${ }^{8}$ RODA PEÑA, José: "José de Guillena y el antiguo retablo mayor de la Capilla de San Andrés de Sevilla”, Laboratorio de Arte, 24, 2012, pp. 351-375. En este artículo podemos apreciar un exhaustivo análisis de los tres retablos mayores que presidieron la capilla, siendo el más antiguo de ellos suscrito bajo contrato en 1564 entre Diego Sánchez, albañil y prioste del hospital de San Andrés, y el pintor Luis de Valdivieso, quien se comprometió a ejecutar las tres tablas principales del retablo, además de las correspondientes al banco.

9 ADPS., Sección Hospitales y centros benéficos, Hospital del Amor de Dios, Legajo 1, expediente de reducción del "Hospital de San Andrés de los albañies", sin foliar. "Cassa del hospital". 
Posteriormente el prioste fue interrogado acerca de la hospitalidad que se llevaba a cabo en dicha institución. El representante del gremio declaró que la única actividad que se realizaba en el hospital era la referente a las juntas de oficiales, además de una fiesta el día de San Andrés, patrón del gremio.

En cuanto a los bienes que poseía la institución, el prioste hizo la siguiente exposición: el dicho hospital tenía una casa, que era la donada por María Pérez, además de una vivienda que se deslindó de la principal para que rentase en alquiler, a fin de poder sufragar los gastos de las remembranzas que los cofrades estaban obligados a hacer cada año, según había dejado en testamento la donante. La vivienda era concedida de por vida a sus inquilinos, además de un heredero, por precio de cuatro mil quinientos maravedíes al año.

Además de estos ingresos, la institución contaba con mil trescientos treinta y nueve maravedíes de tributo al año, que había donado Luisa Núñez de Cabrera, a cargo de las rentas de un "olivar" que estaba situado en el término de Villanueva del Ariscal. Dicha donación ascendía a trescientos ochenta "pies de olivar" y otras tierras que contaban con unos doscientos cincuenta ejemplares de olivo. La escritura de donación estaba firmada ante el escribano público de Sevilla Diego Gabriel en 1579 .

Otro de los capítulos de ingresos eran los referentes a las limosnas de los cofrades, los cuales dejaban cada año unos siete mil maravedíes que daban voluntariamente al hospital, sacados de los remates de las obras en las que participaban, para la asistencia a cofrades que habían caído en la mendicidad. Finalmente el prioste declaró que el inquilino de la casa deslindada del hospital pagaba cada año un tributo de seis gallinas, por las cuales daba cuarenta reales.

Finalmente, Martín Sánchez, prioste de la corporación, hizo un alegato en defensa de la no reducción del hospital de San Andrés, que estaba llamado a integrase en el del Amor de Dios. Para su defensa declaró la propiedad de la casa gremial u hospital "de tiempo inmemorial" y que fue donada por María Pérez mediante testamento en ocho de mayo "era de mill y quatrocientos y uno que fue año del nasimyento de mill y trezientos y sesenta y tres". Las condiciones de dicho testamento establecían que una vez que hubiese muerto su heredero, "Pedro Moso", si no había tenido hijos, la casa pasaría a formar parte de las posesiones del gremio de albañilería, como así fue, debiendo hacerse una serie de misas cada año en memoria de la donante. Los cofrades fundaron la casa hospital, edificando una capilla junto a ella con cargo al trabajo de los propios albañiles, que la fueron manteniendo sin coste a base de su oficio. Ello posibilitó su mantenimiento con las bajas rentas que recibían, únicamente doce ducados y seis gallinas, fruto del arrendamiento de la vivienda que deslindaron del edificio principal. Estas rentas se empleaban en las fiestas celebradas el día de San Andrés, "con visperas misa y sermon y musica y mucha cleresia y cera y colgaduras y toda la solenidad que 
pueden"10. Y al domingo siguiente estaban obligados a celebrar las remenbranças en recuerdo de la donante, María Pérez, a las cuales los cofrades debían asistir con su cera, además de celebrar los entierros de los hermanos y familiares. En ese momento se alegó que en esta casa se elegían "por costumbre muy antigua y hordenanças desta ciudad los alcaldes alarifes y exsaminadores de los albañiles", señalando que a esta ceremonia debían asistir un caballero veinticuatro designado por el Cabildo de la ciudad además de un jurado que diese fe de la elección. De lo expuesto anteriormente fueron presentadas escrituras originales, algo nada frecuente entre las fundaciones hospitalarias sevillanas, que finalmente fueron concentradas en instituciones de mayor entidad.

Por último, el prioste de la corporación expone las razones por las cuales el hospital de San Andrés no debía ser reducido, ya que en este "se hace todo aquello para lo que fue fundado", es decir, labores gremiales y no hospitalarias. Y no se pagaba salario alguno, excepto la vivienda del casero, medio ducado al mes para el mantenimiento de la limpieza de la casa, el aceite para las luminarias, y el socorro de cofrades necesitados.

No quisiéramos abandonar estas líneas sin efectuar algunas reflexiones de lo que supone el dato inédito de la fecha de fundación del hospital de San Andrés de los albañiles. En primer lugar, si damos por cierto el dato del testamento de María Pérez, que legaba una casa para la fundación del hospital del gremio de albañilería, como así parece estar demostrado en el momento de la reducción, mediante la muestra de la escritura original, la fecha de 1401 parece referirse a la era hispánica, con lo que la verdadera fecha de fundación debería establecerse hacia 1363 del calendario gregoriano. Esto supondría un hecho de singular importancia, ya que se trataría de una de las fundaciones gremiales más antiguas de la ciudad. No debemos olvidar que el hospital de San José de los carpinteros, fue fundado en 1578, más de dos siglos después.

Además, podemos establecer una reflexión paralela acerca de esta fundación. Si tomamos en consideración la temprana fecha de fundación de la casa gremial del oficio de la albañilería, vinculada, como parece demostrarse, al culto cristiano y teniendo en cuenta lo que sucedía en otras ciudades donde la albañilería estaba en manos de dos gremios paralelos, uno para cristianos viejos y otro para mudéjares, como es el caso de Granada y Zaragoza ${ }^{11}$, podríamos estar aquí ante la prueba fehaciente de que en Sevilla existió la separación entre las diferentes religiones de los oficiales dedicados a la profesión de la albañilería, debido a la fuerte vinculación religiosa cristiana que demuestran los documentos aquí expuestos.

Podrían, en todo caso, asaltarnos ciertas dudas de si realmente estamos ante la fundación de la casa gremial de los albañiles sevillanos, o simplemente de una

10 Ibídem.

11 BORRÁS GUALIS, Gonzalo: El Arte Mudéjar. Teruel, 1990, pp. 146-150. Véase también, LÓPEZ GUZMÁN, Rafael: Arquitectura mudéjar. Madrid, 2000, p. 68. 
corporación dedicada exclusivamente a labores asistenciales o de hermandad creada ex profeso para atender a las necesidades religiosas de los oficiales. Estas dudas creemos que pueden quedar disipadas si atendemos a los detalles del texto original donde se especifica claramente que las casas eran donadas para la celebración de los cabildos de oficiales albañiles, así como para la elección de los alcaldes-alarifes, miembros inequívocos de la existencia de un gremio consolidado y jerarquizado, así como para la celebración de los exámenes de maestría, algo sobre lo que sólo la corporación podía ejercer el control.

La fecha de fundación en 1363 resulta de gran importancia por encontrarse sensiblemente cercana al terrible seísmo sufrido por la ciudad en 1356, el cual arruinó la mayoría de edificios parroquiales sevillanos, con lo que debieron acometerse importantes labores de reconstrucción, hecho que propiciaría un inusitado crecimiento del oficio de la albañilería. Fue en ese momento cuando debió sentirse la necesidad de fundar la casa gremial que aglutinase los intereses comunes de estos profesionales de la construcción, que tanta importancia habían tenido en el desarrollo de la arquitectura sevillana.

Fecha de recepción: 30 de septiembre de 2015.

Fecha de aceptación: 20 de noviembre de 2015.

\section{APÉNDICE DOCUMENTAL}

1584. Noviembre, 22. Archivo Diputación Provincial de Sevilla, Sección Hospitales y Centros Benéficos, Hospital del Amor de Dios, legajo I. Expediente de reducción del Hospital de San Andrés de los albañiles, sin foliar. Hemos procurado reproducir el formato que el texto presenta en el archivo.

"Relación del hospital de san andres que se dice de los alvañires en la collacion de sant andres_

En la cibdad de sevilla a veinte y dos dias del mes / de noviembre de mill e quinientos y ochenta y quatro años I parescio presente martín sanchez alvañir prioste que dixo I ser del ospital y cofradia de señor san andres de los alvañi / res que esta sita en la collacion de sant andres desta cibdad non / vrado por el cabildo y cofrades de la dicha cofradia y dixo / que en cumplimiento de lo mandado por el Ilustrisimo cardenal I arçobispo de sevilla con asistencia del señor Licenciado barrio / nuevo de peralta juez de su majestad en lo tocante a la rre / ducion de los ospitales viene a dar cuenta y rrazon de los / bienes rrayzes y muebles y tributos que el dicho ospi / tal tiene con los cargos de misas y remembranzas / y fiestas con que los dexaron y para ello exivió los libros / y escripturas que el dicho ospital tiene lo qual yo / el notario infra scripto por virtud de lo mandado por su / Señoria Ilustrísima en presencia del dicho prioste saque / la rrelación siguiente: 


\section{Cassa del ospital}

E luego el dicho martin sanchez prioste declaro que la cassa que el dicho ospital tiene I esta en la dicha collacion de sant andres / frontero de las casas de Pedro de Pineda I que lindan con casas del mesmo ospital I por la una parte y por la ottra la / casa que esta en la esquina de la / calle de la benera y calle real / La qual dicha casa tiene capilla con / un rretablo y altares en que se I dize misa todas las beces que I se juntan los dichos cofrades en / sus cabildos. El qual dicho rretablo es de pin / cel con ciertas figuras de santos_.

\section{Fundacion}

Luego fue rrecebido juramento en / forma de dicho por Dios y la cruz del dicho martin / sanchez prioste y abiendole fecho le fue mandado / declare quien fundo el dicho ospital / y muestre la fundaçión el qual dijo que el / dicho hospital lo dejo maria perez mujer / que fue de diego fernandez como consta / de su testamento que yxsibio escrito en perga / mino su fecha en Sevilla a ocho días del / mes de mayo del año de mill y quatro / cientos y un año signado de gomez pe / rez escribano publico de sevilla por el qual pareze I dejo a los albanires desta ciudad la dicha / cassa para el dicho hospital a donde I se juntasen a nombrar y elejir a / larifes y alcaldes del dicho oficio desta I ciudad y por ello los dichos cofrades / en cada un año le hazen una rre / menbrança en la dicha iglesia de I san andres con sus misas conforme I al dicho testamento_/

\section{Ospitalidad}

Preguntado que hospitalidad se / haze en el dicho hospital dijo que non / hazen ninguna mas de la dicha jun / ta y una fiesta el día de señor / san andres de su boluntad y no / por obligación que tengan_/

Y que la hazienda que tienen es / la siguiente_/

$$
N^{o}-1
$$

- Tiene el dicho hospital la dicha cassa de I suso declarada y deslindadada y una / casilla que se saco de la dicha cassa pa I ra que rrentase para hazer las dichas / Remenbranças de suso declarada I que tienen de obligacion y la fiesta / boluntaria el dia del santo / la qual dicha casilla la dicha cofradia / tiene dada de por bida a luis cabello I del castillo sastre y ana de torralba / su mujer vecinos desta ciudad por las / bidas de los suso dichos y de un heredero / por precio y cuantia de quatro mill / y quinientos maravedies en cada un año como cons / ta de la escritura que ysibio signa / da de gaspar de toledo escribano publico de sevilla / su ffecha en sevilla beinte y ocho dias I del mes de julio de mill y quinientos y / sesenta y tres años_ 


\section{$N^{0}-2$}

- Yten tiene el dicho hospital mill y tre / cientos y treinta y nueve mrs. que dejo / al dicho hospital de tributo en cada un / año luisa nuñez de cabrera doncella / sobre una suerte de olibar que es en termino / de billanueba de ariscal que se dize / Rui diez en que puede aber trecien / tos y ochenta pies de olibar poco mas / o menos que lindan de la una parte / con olibar de la bicaria de la dicha billa / y de la otra con olibar de las mon / jas de san clemente desta ciudad_

Y sobre otro pedazo de olibar en el dicho / termino que se dize bia arroyo que pue I de aver docientos y cincuenta pies poco / mas o menos que lindan con oliba / res de la fabrica de la yglesia de / la dicha billa de los quales dichos mill I trecientos y treinta y nuebe mrs. I hizo donación al dicho hospital como / consta de la dicha escritura que I parece passo ante diego graviel escribano / público de sevilla su fecha en diez y seis de / março de mill y quinientos y setenta y nue / be años los quales dichos mrs. del dicho / tributo faga por escritura de rre / conocimiento ysabel martinez brabo / mujer que fue de pero hernandez de agüero / difunto como consta de la dicha I escritura de rreconocimiento que pa / rece paso ante el dicho diego grabiel / su fecha en beinte y ocho de mayo / de mill y quinientos y ochenta y un años / y no tiene cargo ni obligación ninguno.

\section{$N^{0}-3$}

Yten declaro el dicho prioste que I se dejan de limosna en cada un año siete I mill mrs. que dan de su boluntad / los cofrades de la dicha cofradia que en / trellos de los rremates de obras que / se hazen de su oficio de albañires / se aplican para probes cofrades / que tengan necesidad y esto es bolun / tario_

$$
N^{o}-4
$$

Yten el dicho Prioste declaro I que se pagan a la dicha cofradia I en cada un año seis gallinas / por el dicho Luis cabello como tenedor / de las dichas casas del dicho hospital por / las quales da quarenta rreales en dineros por... a nos..._

\section{Ilustrisimos}

martin sanchez como mayordomo del hospital y cofra / dia de san andres de los albañies en la collacion de san andres con / protestacion de no consentir en comision que aya expirado / ni tomar la defensa desta caussa mas de si y en quanto / sea obligado agora que me fue dado treslado de los brebes / de nuestro muy santo padre pio quinto de felice rrecordacion y / cocnision de su majestad y notificado auto de 
vuestra Ilustrisima para / la rreducion de los hospitales digo que en ella no es con I prehendido el dicho hospital porque es casa de la dicha I cofradia la qual cofradia es fundada de tiempo ynme / morial y ubo la dicha cassa de mayor perez que hizo su I testamento en ocho de mayo era de mill y quatrocientos / y uno que fue año del nasimyento de mill y trezientos y sesenta y tres y dexo por su heredero a pedro moso I y que si muriese sin hijos susediese la dicha cofradia / de san andres en su herencia y fue ansi que sucedio y / les fueron adjudicados a la dicha cofradia las dichas casas I con cargo de una rremenbrança a la dicha mayor / perez y los dichos cofrades fundaron el dicho hospital / en la dicha cassa y la edificaron y labraron en el a lo que I llaman yglesia con su rretablo y altar y alli junto / labraron otra fiesta para sus cabildos y por lo / alto desta piesa del cabildo esta doblado para bi / bienda del casero y como heran albañires lo labra I ron a costa de su trabaxo y ansi lean sustentado I sin tener como no tienen de rrenta sino doze ducados / y seis gallinas que les rrenta una casilla que sa / caron del dicho hospital y estos gastan en una I fiesta que hazen en el mesmo hospital dia de san / andres con visperas misa y sermon y musica y / mucha cleresia y cera y colgaduras y toda la / solenidad que pueden y el domingo adelante / hazen rremenbrança con visperas y misa can / tada en san andres por la dicha mayor perez y todos / los cofrades asisten con su cera y mas hazen los / entierros de los hermanos y sus mujeres e hijos / y en la dicha cassa se eligen por costumbre muy antigua / y hordenanças desta ciudad los alcaldes alarifes y exsaminadores de los albañies y a esta elesion a / sisten un veinte y quatro y un jurado como consta / por titulos y de lo de arriba dicho tienen originales / de manera que esta casa es suya de la dicha cofradia / y en ella se haze todo aquello para que fue fundada / y quitandola se perderian las dichas obras las qua / les con la dicha cofradia podian yr en cresimiento y si notoriamente no es este de los ospitales y cassas / que se mandan rreduzir ni dan salario ninguno / sino al casero no mas que un aposento en que vivir / porque guarda la dicha casa y la tiene linpia para / las dichas selecsiones y cabildos y medio ducado se le I da cada mes para azeite a la lanpara y por po / der socorrer a cofrades que bienen a pobreza y a oficiales / albañies que bienen tanbien a nesesidad tiene por I costumbre de quando salen al rremate algunas / obras lo que los duenos de las tales hobras dan porque I se junten los dichos albañies a tomar la dicha hobra lo I dexan todos ellos para el hospital y obras que / los dichos cofrades hazen _ y todo esto cesaria / si el dicho hospital se rredujese y finalmente sesan / con el todas las causas de rreducion y militan las / contrarias para no hazerla_/

Por tanto pido y suplico a vuestra Ilustrisima declare no poder I ni aver lugar rreduzir el dicho hospital casa y bienes de la otro alguno ni mudar sus obras alguna / ni a otra parte y lugar haziendo lo que mas en fabor sea I del dicho hospital y cofradia $y$ de la rrepublica desta / ciudad sobre que pido cumplimiento de justicia y rrigor perjudicial no me ofreciese siendo a cossa demasiada / para ello estas."

LABORATORIO DE ARTE 28 (2016), pp. 103-112, ISSN 1130-5762

e-ISSN 2253-8305 - DOI http://dx.doi.org/10.12795/LA.2006.i.01.05 